\title{
Adherence to infection prevention measures in a statewide spleen registry
}

\section{Julie Wang \\ MBBS, BMedSci Haematology Registrar? \\ Penelope Jones \\ RN, GradDipEpi Manager, Victorian Spleen Registryl \\ Allen C Cheng MBBS, FRACP, PhD Associate Professor 1,2 \\ Karin Leder MBBS, FRACP, PhD Associate Professor \\ 1 Alfred Hospital, Melbourne, VIC \\ 2 Department of Epidemiology and Preventive Medicine, Monash University, \\ Melbourne, VIC. \\ p.jones@alfred.org.au}

MJA 2014; 200: 538-540 doi: 10.5694/mjal3.10630
P eople who have had a splenectomy or who have hyposplenia have a greater than 50 -times higher risk of developing serious infection with encapsulated organisms than the general population. ${ }^{1}$ Although it occurs rarely (estimated lifetime risk, $5 \%$ ), overwhelming postsplenectomy infection (OPSI) is associated with a mortality of $40 \%$ to $70 \% .^{2}$ Although the risk of OPSI is highest in the first few years after splenectomy, cases have occurred 10 to 40 years afterwards, suggesting the risk is lifelong. ${ }^{3,4}$

The high mortality associated with OPSI has led to guidelines for its prevention, although previous studies have found poor adherence to preventive measures. ${ }^{4-8}$ The Victorian Spleen Registry (VSR) was established in 2003, and is the only registry in Australia of patients who have undergone splenectomy or who have hyposplenia. The VSR provides an educational kit on registration and an annual vaccination reminder. The current recommendations provided to patients are described below.

- Education: awareness of the risk and preventive measures, and a clear action plan for febrile illness, animal bite or planned overseas travel.

- Vaccination: currently recommended are both the 23-valent pneumococcal polysaccharide vaccine and the 13-valent pneumococcal conjugate vaccine, the Haemophilus influenzae type b conjugate vaccine, the 4 -valent meningococcal conjugate vaccine; and an annual seasonal influenza vaccine. Specific recommendations vary by age. ${ }^{9}$

- Antibiotic prophylaxis: lifelong antibiotics are recommended for immunosuppressed patients, and for at least 2 years after splenectomy for all other patients. Further, patients are advised to keep an emergency supply of antibiotics in the event of a febrile illness.

The aim of our study was to assess self-reported adherence to health maintenance behaviour and infection

\section{Abstrac}

Objective: To assess self-reported adherence to measures for preventing infection in patients registered in the Victorian Spleen Registry (VSR).

Design, participants and setting: Cross-sectional survey in May 2010 of all patients who had been registered on the VSR for at least 5 months, were able to speak English, and were not living in an institution.

Main outcome measures: Rates of prophylactic antibiotic use, having an emergency supply of antibiotics available, receipt of any recommended booster vaccination (in patients at $>5$ years since splenectomy) and receipt of 2009 influenza vaccination.

Results: 1175 patients were sent questionnaires, of whom 889 (75.7\%) responded. Self-reported adherence to taking prophylactic antibiotics was lower with time since splenectomy ( $82.9 \%$ for $<2$ years since splenectomy, $27.4 \%$ for $\geqslant 30$ years), as was having an emergency supply of antibiotics available ( $74.4 \%$ for $<2$ years and $60 \%$ for $\geqslant 30$ years since splenectomy). The proportion receiving the seasonal influenza vaccine and recent booster vaccines (for those at $>5$ years since splenectomy) was high. Of patients registered for more than 12 months, $37.0 \%$ reported an infection requiring additional antibiotics within the prior year, and $26.1 \%$ of these required hospitalisation (including one with a case of overwhelming postsplenectomy infection [OPSI]). Use of prophylactic antibiotics was inversely associated with the rate of infections requiring additional antibiotic therapy (odds ratio, 0.75; 95\% Cl, 0.57-0.98; $P=0.036$ ).

Conclusions: The proportion of VSR registrants adhering to current postsplenectomy guidelines was higher than rates reported elsewhere, and over a third reported infections requiring a course of antibiotics within the prior 12 months. However, only one OPSI occurred. These results support the view that a spleen registry can promote health maintenance behaviour in asplenic patients, which is likely to help prevent serious infections.

rates in patients with asplenia or hyposplenia who are registered with the VSR. We hypothesised that patients registered with the VSR would report higher adherence to and awareness of health maintenance behaviour than would other populations of patients with asplenia or hyposplenia reported in the literature, who were not part of a splenectomy registry.

\section{Methods}

All Victorian patients with a clinical diagnosis of asplenia or hyposplenia are eligible for enrolment on the VSR. A small number of patients from outside Victoria are also included. Patients are identified either retrospectively (if they have a history of prior splenectomy) or prospectively (from hospital-based surveillance). On enrolment, patients are contacted by phone and an education kit is sent with a personalised vaccine report. Details of the reason for the splenectomy and vaccinations given are confirmed with the hospital, general practitioner and/or patient. Reminders are sent to GPs and patients when booster vaccines are due, and patients are reminded that influenza vaccination is recommended in an annual newsletter.

In May 2010, a cross-sectional survey was mailed to all English-speaking VSR registrants who had been registered for more than 5 months, and who were living at home (not in a nursing home or prison). Patients who did not respond were contacted by telephone and either were sent a repeat questionnaire by mail, or completed the survey over the telephone.

We compared measures of selfreported adherence (use of prophylactic antibiotics, availability of emergency antibiotics, receipt of any of the recommended booster vaccinations if it was more than 5 years since the splenectomy, receipt of the 2009 influenza vaccine) with time since splenectomy. To explore whether registrants may have been self-selected according to health maintenance behaviour, we compared similar measures in registrants who were prospectively enrolled ( $<12$ months since splenectomy), with those retrospectively 
1 Characteristics of respondents and non-respondents to a survey of Victorian Spleen Registry registrants in May 2010

\begin{tabular}{|c|c|c|c|}
\hline Characteristic & Respondents & Non-respondents & $P$ \\
\hline Total number & 889 & 286 & \\
\hline Age* & & & $<0.001$ \\
\hline$<18$ years & $12(1.4 \%)$ & $6(2.1 \%)$ & \\
\hline 18 to $<40$ years & $176(20.0 \%)$ & $113(39.5 \%)$ & \\
\hline $40-65$ years & 449 (50.9\%) & $117(40.9 \%)$ & \\
\hline$>65$ years & $245(27.8 \%)$ & $50(17.5 \%)$ & \\
\hline Sex & & & $0.013^{\dagger}$ \\
\hline Male & $441(49.6 \%)$ & $166(58.0 \%)$ & \\
\hline Duration of asplenia ${ }^{\ddagger}$ & & & $<0.001^{5}$ \\
\hline$<2$ years & $199(22.8 \%)$ & $67(25.5 \%)$ & \\
\hline $2-<5$ years & $171(19.6 \%)$ & $70(26.6 \%)$ & \\
\hline $5-<10$ years & 115 (13.2\%) & $44(16.7 \%)$ & \\
\hline $10-<30$ years & $199(22.8 \%)$ & $57(21.7 \%)$ & \\
\hline$\geqslant 30$ years & $190(21.7 \%)$ & $25(9.5 \%)$ & \\
\hline Duration registered & & & $<0.65^{\S}$ \\
\hline$<1$ year & $155(17.6 \%)$ & $51(17.8 \%)$ & \\
\hline $1-<2$ years & $324(36.7 \%)$ & $98(34.3 \%)$ & \\
\hline $2-<3$ years & $140(15.9 \%)$ & $41(14.3 \%)$ & \\
\hline$\geqslant 3$ years & $264(29.9 \%)$ & $96(33.6 \%)$ & \\
\hline Reason for splenectomy & & & $<0.001^{\dagger}$ \\
\hline Trauma & $291(32.7 \%)$ & $136(47.6 \%)$ & \\
\hline Haematological disorder & $258(29.0 \%)$ & $71(24.8 \%)$ & \\
\hline Malignancy & $125(14.1 \%)$ & $27(9.4 \%)$ & \\
\hline Unplanned splenectomy** & 99 (11.1\%) & 26 (9.1\%) & \\
\hline Other & 116 (13.1\%) & 26 (9.1\%) & \\
\hline
\end{tabular}

* Data missing for seven respondents. $\dagger \chi^{2}$ test. $\ddagger$ Data missing for 15 respondents and 23 nonrespondents. \$Mann-Whitney $U$ test. \$ Data missing for six respondents. **Complication resulting from other surgery.

identified (enrolled >12 months after splenectomy).

The activities of the VSR are covered by Alfred Health Human Research Ethics Committee (approval number HREC 189/07).

\section{Statistical analysis}

We tested the null hypothesis that there were no differences between groups in non-parametric continuous variables using the Mann-Whitney $U$ test and in proportions using the $\chi^{2}$ test. A logistic regression model examined factors associated with selfreported infection within the previous 12 months, and included variables that were associated with infection on a univariate analysis with a significance level of $P<0.2$ in a stepwise selection process. We rejected the null hypothesis at a significance level of $P<0.05$. Statistical procedures were performed using Stata statistical software, version 10 (StataCorp).

\section{Results}

In May 2010, there were 1304 people were sent questionnaires, of whom 889 (75.7\%) responded. Box 1 shows the demographic characteristics of respondents and non-respondents. Non-respondents were more likely to be male $(P=0.013)$ and younger (median age, 44 versus 55 years for responders; $P<0.001)$.

A higher proportion of patients prospectively enrolled in the VSR $(<12$ months after splenectomy) reported having an emergency supply of antibiotics available than those who were enrolled longer after their splenectomies $(72.3 \%$ v $61.3 \% ; P=0.001)$. The proportions reporting being vaccinated enrolled in the VSR. Of these, 1175 for 2009 seasonal influenza were similar $(82.7 \%$ v $85.2 \% ; P=0.67)$, as were the proportions reporting receiving 2009 H1N1 monovalent vaccine $(58.3 \%$ v 63.1\%; $P=0.07)$.

Self-reported adherence to prophylactic antibiotic therapy was highest in patients who had recently undergone splenectomy, for whom daily oral antibiotics are most strongly recommended (Box 2). The proportion of patients who had an emergency supply of antibiotics available became lower with time since splenectomy. Self-reported rates of receiving any booster vaccinations (in patients at $>5$ years since splenectomy), and 2009 seasonal influenza vaccination remained high.

Of survey respondents registered for more than 12 months, $37.0 \%$ reported an infection requiring additional antibiotic therapy within the prior year and, of these, $26.1 \%$ required hospitalisation; this included one case of OPSI. Indication for splenectomy, age at splenectomy and time since splenectomy were not associated with the risk of infection. The use of prophylactic antibiotic therapy at the time of the infection appeared to be inversely associated with infection requiring additional antibiotic therapy (odds ratio, 0.75; 95\% CI, 0.57-0.98; $P=0.036)$. No factors were identified as being associated with infection resulting in a need for hospitalisation.

Overall, $80.8 \%$ of patients recalled having received educational material about the risk of infection since their splenectomies. This material comprised: an education kit from the VSR (63.3\%); an education kit from a hospital doctor (12.1\%); education from a GP (29.8\%); other education from the VSR (22.6\%); other sources of education (4.8\%). Time since registration was not associated with education received.

\section{Discussion}

In this survey of registrants of a statewide spleen registry in Victoria, we found high adherence to current postsplenectomy guidelines, including using prophylactic antibiotics, keeping an emergency supply of antibiotics, and receiving booster vaccinations. The proportion of patients taking prophylactic antibiotics and who had an available emergency supply of antibiotics was inversely correlated with time since splenectomy. However, most 


\section{Health maintenance behaviour among Victorian Spleen Registry registrants by duration of asplenia}

\begin{tabular}{|c|c|c|c|c|c|}
\hline \multirow[b]{2}{*}{ Health maintenance behaviour } & \multicolumn{4}{|c|}{ Duration of asplenia (years) } & \multirow[b]{2}{*}{$P$} \\
\hline & $<2$ & 2 to $<10$ & 10 to $<30$ & $\geqslant 30$ & \\
\hline Number of patients & 199 & 286 & 199 & 190 & \\
\hline Taking antibiotics prophylactically & $165(82.9 \%)$ & $181(63.3 \%)$ & $64(32.2 \%)$ & $52(27.4 \%)$ & $<0.001$ \\
\hline Emergency antibiotics available & $148(74.4 \%)$ & $196(68.5 \%)$ & 119 (59.8\%) & $114(60.0 \%)$ & 0.001 \\
\hline \multicolumn{6}{|l|}{ Vaccinations } \\
\hline Any booster since splenectomy & na & $50 / 65 *(76.9 \%)$ & $111 / 135(82.2 \%)$ & $92 / 120(76.7 \%)$ & 0.86 \\
\hline 2009 seasonal influenza TIV & $163(81.9 \%)$ & $243(85.0 \%)$ & $162(81.4 \%)$ & $166(87.4 \%)$ & 0.40 \\
\hline HIN1 2009 influenza & $128(64.3 \%)$ & $163(57.0 \%)$ & 114 (57.3\%) & $127(66.8 \%)$ & 0.22 \\
\hline
\end{tabular}

patients at less than 2 years after splenectomy, for whom antibiotic prophylaxis is most strongly recommended, were taking prophylactic antibiotics. A significant proportion of respondents reported receiving booster vaccinations and annual influenza vaccinations. However, we did not ascertain the date of receipt of booster vaccinations, so the proportion of patients who received booster vaccinations on time is not known.

Overall, the proportions of patients in our study who displayed health maintenance behaviours as recommended by current guidelines were higher than those in other studies. ${ }^{5-10}$ A study of 62 splenectomy patients in the New South Wales Hunter area found only $11.3 \%$ of patients (7/62) had an emergency supply of antibiotics, and only one patient recalled receiving prophylactic antibiotics for 1 year after surgery. ${ }^{8}$ In a study of 536 patients in the Netherlands, only $14.4 \%$ of patients (77/536) received any prophylactic antibiotics after surgery, and $7.6 \%$ of patients $(41 / 536)$ had an emergency supply of antibiotics. ${ }^{10}$

Somewhat unexpectedly, we found a high proportion of patients who reported having an episode of infection for which they required a course of antibiotics within the prior 12 months. While many of these episodes are likely to represent minor or viral infections, a significant proportion of these patients reported being hospitalised for infection. We could not determine the nature or severity of infections, and this finding may relate to the successful education of VSR registrants about the importance of ensuring appropriate and rapid medical attention when patients are unwell. In contrast to other studies, we did not find haematological disorders, age or recent splenectomy to be risk factors for self-reported infection. ${ }^{1,11-13}$ This may reflect a true lack of difference in infection rates or a lack of statistical power. Further work is required to characterise these infections and determine whether or not they were related to asplenia. As of 2012 , we were aware of only one occurrence of OPSI leading to intensive care admission among the 2574 VSR registrants at that time (representing tion), which compares favourably with reported rates of OPSI of around one in 200 patient-years. ${ }^{5}$

There are several limitations to our study. We achieved a reasonable response rate $(75.7 \%)$ from those registrants who were surveyed, but the validity of our findings may be limited by responder bias. We did not verify antibiotic use or vaccination status, which were self-reported. While registration with the VSR may be a marker of other health maintenance behaviours, this is less likely to be the case for prospectively enrolled patients who were routinely registered at the time of splenectomy. Other than the availability of an emergency supply of antibiotics, we did not find adherence to preventive measures to be different in prospectively enrolled patients compared with retrospectively enrolled patients. We did not verify the type or timing of booster vaccines, and over 5500 patient-years of observa- therefore cannot assess full compliance with guidelines.

Previous studies suggest that patient registries for individuals with asplenia are cost-effective based on modelled outcomes. ${ }^{14}$ Although further work is required to characterise the nature of frequently reported infections other than OPSI in this patient group, our results suggest that providing educational material and regular reminders to patients effectively promotes adherence to recommended measures, which is likely to help prevent serious infections in patients with asplenia.

Competing interests: Allen Cheng has received research funding to assist with an influenza vaccine safety study from CSL Ltd. Karin Leder has received research funding (unrelated to this manuscript) from GlaxoSmithKline (GSK), and travel support from GSK and Sanofi Pasteur. Received 12 May 2013, accepted 20 Feb 2014.

1 Hansen K, Singer DB. Asplenic-hyposplenic overwhelming sepsis: postsplenectomy sepsis revisited. Pediatr Dev Pathol 2001; 4: 105-121.

2 Lynch AM, Kapila R. Overwhelming postsplenectomy infection. Infect Dis Clin North Am 1996; 10: 693-707.

3 Melles DC, de Marie S. Prevention of infections in hyposplenic and asplenic patients: an update. Neth J Med 2004; 62: 45-52.

4 Waghorn DJ. Overwhelming infection in asplenic patients: current best practice preventive measures are not being followed. $J$ Clin Pathol 2001; 54: 214-218.

5 El-Alfy MS, El-Sayed MH. Overwhelming postsplenectomy infection: is quality of patient knowledge enough for prevention? Hematol J 2004; 5: 77-80.

6 O'Donnell J, McGreal G, Daly P, et al. Management of patients undergoing splenectomy in an Irish teaching hospital: impact of guidelines. Ir J Med Sci 2004; 173 : $136-140$.

7 Hasse B, Moll C, Oehy K, et al. Anti-infectious prophylaxis after splenectomy: current practice in an eastern region of Switzerland. Swiss Med Wkly 2005; 135: 291-296.

8 Wilkes A, Wills V, Smith S. Patient knowledge of the risks of post-splenectomy sepsis. ANZ J Surg 2008; 78: 867-870.

9 The Australian immunisation handbook. 10th ed. Canberra: Australian Government Department of Health, 2013.

10 Lammers AJ, Veninga D, Lombarts MJ, et al. Management of post-splenectomy patients in the Netherlands. Eur I Clin Microbiol Infect Dis 2010; 29: 399-405.

11 Bisharat N, Omari H, Lavi I, Raz R. Risk of infection and death among post-splenectomy patients. J Infect 2001; 43: 182-186.

12 Cullingford GL, Watkins DN, Watts AD, Mallon DF. Severe late postsplenectomy infection. Br J Surg 1991; 78: 716-721.

13 Dendle C, Sundararajan V, Spelman T, et al. Splenectomy sequelae: an analysis of infectious outcomes among adults in Victoria. Med J Aust 2012; 196: 582-586.

14 Woolley I, Jones P, Spelman D, Gold L. Costeffectiveness of a post-splenectomy registry for prevention of sepsis in the asplenic. Aust NZ J Public Health 2006; 30: 558-561. 